\title{
-O) CLINICAL SNAPSHOT
}

\section{Giant Bulla Imitating Tension Pneumothorax}
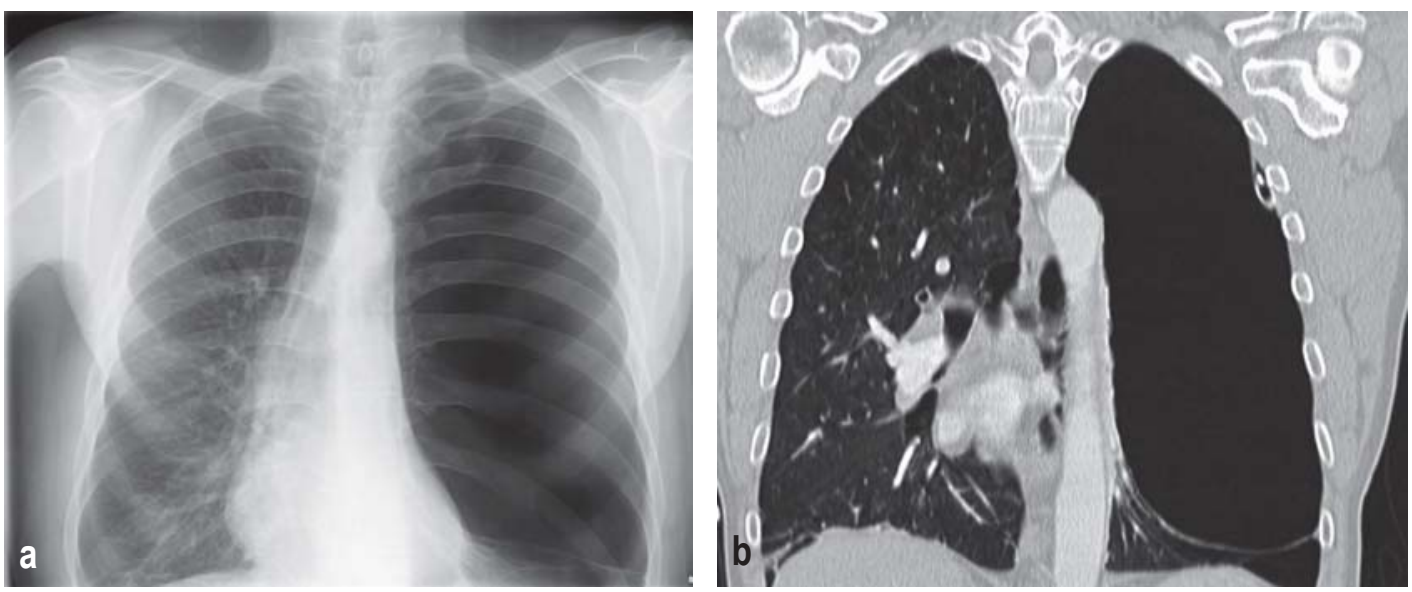

A 61-year-old man with a long history of exercise dyspnea came to the emergency department with acute respiratory distress at rest. His oxygen saturation was below $90 \%$, and there were no breathing sounds on the left side. Posteroanterior chest radiography in expiration with the patient standing erect showed almost complete absence of lung markings in the left thorax with residual lung and what looked like a pneumothorax line in the basal portion, together with displacement of the mediastinum to the right (Figure 1).

We assumed the diagnosis of left tension pneumothorax and inserted a thoracic drain. Repeat radiography showed no change. Computed tomography (coronal thorax-lung window) then revealed a giant bulla (Figure 2). Thoracic drain placement had not perforated the bulla.

The patient's respiratory distress was gradually relieved by means of oxygen administration. Later, elective resection of the bulla took place. The patient was then symptom-free at rest and could perform normal physical activities.

Patients with an extensive history of pulmonary emphysema and conspicuous findings on radiography should, provided their clinical status permits, be examined by thoracic CT for confident differentiation between pneumothorax and bullous lesions.

Dr. med. Matthias Schumann, Ahmad Hamed, Dr. med. Thomas Kohl, Klinik für Thorax- und Gefäßchirurgie, Zentralklinik Bad Berka, matthias.schumann@zentralklinik.de

Conflict of interest statement: The authors declare that no conflict of interest exists.

Translated from the original German by David Roseveare.

Cite this as: Schumann M, Hamed A, Kohl T: Giant bulla imitating tension pneumothorax.

Dtsch Arztebl Int 2019; 116: 461. DOI: 10.3238/arztebl.2019.0461 